\title{
Hydrostatic Pressure Dependence of the Fundamental Bandgap of InN and In-rich Group III-Nitride Alloys
}

\author{
S. X. Li, J. Wu, E.E. Haller, \\ Materials Sciences Division, Lawrence Berkeley National Laboratory, and Department of \\ Materials Science and Engineering, University of California, Berkeley, California 94720 \\ W. Walukiewicz, W. Shan, \\ Materials Sciences Division, Lawrence Berkeley National Laboratory, Berkeley, \\ California 94720 \\ Hai Lu, and William J. Schaff \\ Department of Electrical and Computer Engineering, Cornell University, Ithaca, New \\ York 14853
}

We report studies of the hydrostatic pressure dependence of the fundamental bandgap of $\mathrm{InN}$, In-rich $\operatorname{In}_{1-x} \mathrm{Ga}_{x} \mathrm{~N}(0<x<0.5)$ and $\operatorname{In}_{1-x} \mathrm{Al}_{x} \mathrm{~N}(x=0.25)$ alloys. The bandgap shift with pressure was measured by optical absorption experiments with samples mounted in diamond anvil cells. The pressure coefficient is found to be $3.0 \pm 0.1$ $\mathrm{meV} / \mathrm{kbar}$ for InN. A comparison between our results and previously reported theoretical calculations is presented and discussed. Together with previous experimental results, our data suggest that the pressure coefficients of group-III nitride alloys have only a weak dependence on the alloy composition. The photoluminescence signals appear to yield significantly smaller pressure coefficients than the bandgap from absorption measurements. This is due to emission associated with highly localized states. Based on these results, the absolute deformation potentials of the conduction and valence band edges are estimated.

PACS numbers: 78.66.Fd, 78.20.Ci

Electronic Mail:w_walukiewicz@lbl.gov 
The recent discovery of the $0.7 \mathrm{eV}$ fundamental band gap of $\mathrm{InN}[1-5]$ has extended the range of the direct band gaps of group III-nitride alloys into the near infrared part of the electromagnetic spectrum. In the view of the newly determined $\mathrm{InN}$ band gap it has become necessary to reevaluate the composition dependencies of the major material parameters of group III-nitride alloys. For instance, studies of the composition dependence of the band gaps in In-rich $\operatorname{In}_{1-x} \mathrm{Ga}_{x} \mathrm{~N}$ and $\operatorname{In}_{1-x} \mathrm{Al}_{x} \mathrm{~N}$ alloys have led to new values of the bowing parameters that are much lower than those previously determined by assuming the band gap of $\mathrm{InN}$ to be $1.9 \mathrm{eV}$ [6]. There have been only a few experimental studies on the pressure behavior of $\mathrm{GaN}$ [7-9], Ga-rich $\operatorname{In}_{1-x} \mathrm{Ga}_{x} \mathrm{~N}$ alloys $[8,10]$ and AlN [11]. Although there is a relatively good consensus on the band gap pressure coefficients of GaN and AlN, much less is known about the pressure dependence of the energy gaps in In containing group III-nitride alloys. A wide range of band gap pressure coefficients has been found even in the most extensively studied Ga-rich $\operatorname{In}_{I-}$ ${ }_{x} \mathrm{Ga}_{x} \mathrm{~N}$ alloys $[8,10]$. To our knowledge, there has been no report on hydrostatic pressure studies on the bandgap of $\mathrm{InN}$ and In-rich $\operatorname{In}_{1-x} \mathrm{Ga}_{x} \mathrm{~N}$ and $\operatorname{In}_{1-x} \mathrm{Al}_{x} \mathrm{~N}$ alloys.

In this paper we report results of investigations of the hydrostatic pressure behavior of the fundamental bandgaps of $\mathrm{InN}$, In-rich $\operatorname{In}_{I-x} \mathrm{Ga}_{x} \mathrm{~N}(0<x<0.5)$ and $\operatorname{In}_{I-}$ ${ }_{x} \mathrm{Al}_{x} \mathrm{~N} \quad(x=0.25)$ alloys based on optical absorption and photoluminescence measurements. Together with previous experimental results, our data provide an overall picture of the hydrostatic pressure behavior of the fundamental bandgap of group IIInitride alloys over a large range of compositions.

The InN film used in this study was $7.5 \mu \mathrm{m}$ thick and grown by Molecular-Beam Epitaxy (MBE) on (0001) oriented sapphire with a GaN buffer layer (250 nm). $\operatorname{In}_{1-x} \mathrm{Ga}_{x} \mathrm{~N}$

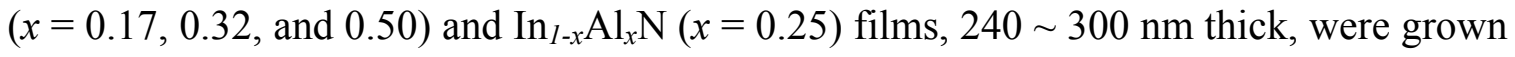
with an AlN buffer layer $(200 \mathrm{~nm})$. The free electron concentration and mobility of the InN film are $3.5 \times 10^{17} \mathrm{~cm}^{-3}$ and $2050 \mathrm{~cm}^{2} / \mathrm{V} \cdot \mathrm{s}$, respectively. The free electron concentrations of the InGaN and InAlN films are a few times $10^{18} \mathrm{~cm}^{-3}$, and the mobilities range between $50 \sim 500 \mathrm{~cm}^{2} / \mathrm{V} \cdot \mathrm{s}$. The details of the growth process have been published elsewhere [12]. X-ray diffraction studies show that high-quality wurtzite-structured epitaxial layers formed with their c-axis perpendicular to the substrate surface. 
The optical absorption measurements were performed using quasi-monochromatic light from a halogen tungsten lamp dispersed by a $0.5 \mathrm{~m}$ monochromator as the light source. An InGaAs photodiode was used as the detector for the $\mathrm{InN}$ and $\operatorname{In}_{0.83} \mathrm{Ga}_{0.17} \mathrm{~N}$ samples and a Ge photodiode was used for the rest of the samples. For the pressuredependent optical studies, the sapphire substrates were mechanically thinned down to $\sim 20$ $\mu \mathrm{m}$, and the samples were cut into small chips $\sim 100 \times 100 \mu \mathrm{m}^{2}$ in size and mounted into gasketed diamond anvil cells for the application of hydrostatic pressure. The pressure medium was a mixture of ethanol and methanol (1:4). The applied pressure was calibrated by the standard method of monitoring the red shift of the ruby R1 photoluminescence (PL) line. For pressure-dependent PL measurements, the PL signals were generated in the backscattering geometry by excitation with the $476.5 \mathrm{~nm}$ line of an argon laser. The emission is dispersed in a $1 \mathrm{~m}$ double-grating monochromator and directed to a liquid nitrogen-cooled Ge detector.

Fig. 1 shows the absorption curves (linear absorption coefficient squared) of the InN sample at hydrostatic pressures up to $69 \mathrm{kbar}$. The absorption edge is determined by extrapolating the nearly linear region down to the base line. The absorption coefficient increases from the base line to $\sim 6000 \mathrm{~cm}^{-1}$ at $0.03 \mathrm{eV}$ above the absorption edge. This rate is comparable to the rate of increase of the absorption coefficient of semi-insulating GaAs: $\sim 9000 \mathrm{~cm}^{-1}$ at $E_{\mathrm{g}}+0.03 \mathrm{eV}$ [13]. The weaker absorption in InN might be due to the lack of excitonic enhancement of the band-edge absorption. The absorption edge shows a strong blue shift from $0.63 \mathrm{eV}$ at ambient pressure to $0.84 \mathrm{eV}$ at $69 \mathrm{kbar}$. The absorption curves shift in a parallel fashion without significant changes in the lineshape, indicating that the high crystal quality is preserved within the range of the applied pressure.

Fig. 2 shows the pressure dependence of the absorption edges of all $\mathrm{InN}$, InGaN, and InAlN samples investigated. The error bars of the absorption data originate in the uncertainty in the extrapolation of the absorption curves down to the base line. For comparison to the absorption data, the PL peak energy is also shown as a function of pressure for $\mathrm{InN}$ and $\mathrm{In}_{0.5} \mathrm{Ga}_{0.5} \mathrm{~N}$. Both the absorption edges and the PL peaks show a linear dependence on the hydrostatic pressure, but the dependence of the PL peaks is much weaker. From a least-square linear fit of the absorption data, the pressure 
coefficient of the bandgap of $\mathrm{InN}$ is found to be $3.0 \pm 0.1 \mathrm{meV} / \mathrm{kbar}$, five times larger than the pressure coefficient of the PL peak $(\sim 0.6 \mathrm{meV} / \mathrm{kbar})$ that has been published previously [3].

The residual strain in the epilayer due to lattice-mismatch to the substrate could have an effect on the pressure behavior. If the nitride film attaches pseudomorphically to the sapphire substrate, which has a larger bulk modulus, the sample could experience a lower effective pressure than applied. In Ref. [3], a correction factor of 1.45 was estimated assuming a coherently strained InN film on sapphire. However, our InN sample $(7.5 \mu \mathrm{m})$ has been shown to be fully relaxed by high-resolution transmission electron microscopy and reciprocal space mapping (RSM). In fact, studies show that in MBE grown InN film on GaN buffer layers the residual strain falls off quickly with thickness $[14,15]$. It has been reported that the InN films are fully relaxed after a few bilayers on $\mathrm{GaN}$ [14] or above $35 \mathrm{~nm}$ on sapphire [16] during growth. The $\operatorname{In}_{1-x} \mathrm{Ga}_{x} \mathrm{~N}$ and $\operatorname{In}_{1-x} \mathrm{Al}_{x} \mathrm{~N}$ films grown on AlN buffer layers are also expected to be fully relaxed based on RSM measurements performed on selected samples. This is not surprising considering the large film thickness ( $>240 \mathrm{~nm}$, which corresponds to $>800$ bilayers in c-axis) and the large lattice mismatch between the AIN buffer layers and the In-rich nitride alloys. Therefore, the strain effect of the films will be neglected in the following discussion.

The pressure coefficients are summarized in Fig. 3 for InN, AlN, GaN and their ternary alloys. The data include our work and previous reports of the pressure coefficients from theoretical calculations $[17,18]$ and experiments $[3,8,10,11]$. As seen in Fig. 3, the pressure coefficients of the bandgap of $\operatorname{In}_{1-x} \mathrm{Ga}_{x} \mathrm{~N}$ alloys are very closed to that of pure $\mathrm{InN}$ and fall in between the theoretical predictions by Wei et al. [17] and Christensen et al. [18]. Considering the pressure coefficient of $\mathrm{GaN}$ to be $\sim 4 \mathrm{meV} / \mathrm{kbar}$ [8], the pressure coefficient of the $\operatorname{In}_{1-x} \mathrm{Ga}_{x} \mathrm{~N}$ alloy system increases with increasing $\mathrm{x}$ at a low rate of about $0.01 \mathrm{meV} / \mathrm{kbar}$ per \% Ga mole fraction. The pressure coefficient of $\operatorname{In}_{0.75} \mathrm{Al}_{0.25} \mathrm{~N}$ is closer to the calculation of Christensen and Gorczyca [18] in value, but the trend (slope) of the pressure coefficient with respect to increasing $\mathrm{Al}$ composition shows a better agreement with the calculations of Wei and Zunger [17]. The slope extrapolates to a value of $\sim 5 \mathrm{meV} / \mathrm{kbar}$ at $\mathrm{x}=1$ that is very close to the pressure coefficient of AlN determined from absorption experiments [11]. The pressure coefficient for the InAlN 
system thus has a composition dependence of about $0.02 \mathrm{meV} / \mathrm{kbar}$ per $\%$ of $\mathrm{Al}$ fraction. The small magnitude of the pressure coefficients of group III nitrides as compared to other III-V compounds (for example the GaAs pressure coefficient is $\mathrm{dE}_{\mathrm{g}} / \mathrm{dP}=11$ $\mathrm{meV} / \mathrm{kbar}[19]$ ) can be attributed to their higher ionicity (GaAs Phillips ionicity $f_{\mathrm{i}}=0.31$ while $\left.\mathrm{GaN} f_{i}=0.50[20]\right)$. It has been found that in group III-V semiconductors, higher ionicity leads to smaller pressure coefficients [21]. The trend applies to group-III nitrides, among which larger cations give higher ionicity $\left(\operatorname{AlN} f_{\mathrm{i}}=0.449, \operatorname{GaN} f_{i}=0.500\right.$, and $\operatorname{InN}$ $f_{i}=0.578$ [20]) and thus smaller pressure coefficients.

The large difference between the pressure coefficients of the absorption edge and the PL peak shows the limitation of pressure studies using PL spectroscopy. Similar differences, previously reported in PL studies of Ga-rich $\operatorname{In}_{1-x} \mathrm{Ga}_{x} \mathrm{~N}$ alloys $[8,10]$, are shown in Fig. 3. In Ref. [10], the influence of built-in biaxial strain and quantum confined Stark effect due to an internal piezoelectric field were proposed to explain the smaller PL pressure coefficient in cubic InGaN. However, neither hypothesis can explain the small PL pressure coefficient in pure InN. A small pressure dependence of the PL signal suggests that the photon emission involves a radiative recombination associated with highly localized states. Localized states are a superposition of states from the entire Brillouin zone and exhibit much weaker pressure dependences than the conduction band states at the $\Gamma$ point $[22,23]$. On an absolute energy scale, the energy levels of such states are expected to be constant [24,25]. This assertion is confirmed by results shown in Fig. 4, which display the PL peak energies of $\operatorname{In}_{1-x} \mathrm{Ga}_{x} \mathrm{~N}$ alloys as a function of $\mathrm{Ga}$ composition on an absolute energy scale. The absolute energy scale is plotted by accounting for the valence band offset between $\mathrm{InN}$ and $\mathrm{GaN}$, which has been determined to be $1.05 \mathrm{eV}$ by X-ray photoemission spectroscopy [26]. As seen in Fig. 4, the PL peak energy is essentially independent of the alloy composition and the conduction band minimum (CBM). The energy level of the localized states is close to the CBM in InN. If we assume that the localized levels are independent of pressure and that the slow increase of the PL peak is due to the downward shift of the valence band maximum (VBM), the absolute pressure coefficients for the CBM and the VBM of InN are $d E_{d} d d P=2.4$ $\mathrm{meV} / \mathrm{kbar}$ and $d E_{v} / d P=-0.61 \mathrm{meV} / \mathrm{kbar}$ respectively. Based on the bulk modulus of $\mathrm{InN}$ of $1480 \mathrm{kbar}$ [27], the absolute deformation potentials are $d E_{c} / d \ln V=-3.6 \mathrm{eV}$ and 
$d E_{v} / d \ln V=0.92 \mathrm{eV}$ respectively. The above values are larger than those calculated in Ref [17] $\left(d E_{c} / d \ln V=-2.02 \mathrm{eV}\right.$ and $\left.d E_{v} / d \ln V=0.73 \mathrm{eV}\right)$. Similarly, from our results the absolute deformation potentials of $\operatorname{In}_{0.5} \mathrm{Ga}_{0.5} \mathrm{~N}$ are estimated to be $d E_{c} / d \ln V=-4.3 \mathrm{eV}$ and $d E_{v} / d \ln V=1.4 \mathrm{eV}$.

In conclusion, we have presented results of hydrostatic pressure studies of the fundamental bandgap of InN, In-rich $\operatorname{In}_{l-x} \mathrm{Ga}_{x} \mathrm{~N}(0<x<0.5)$ and $\operatorname{In}_{l-x} \mathrm{Al}_{x} \mathrm{~N}(x=0.25)$ alloys. The pressure dependences of the bandgaps are measured by optical absorption spectroscopy in a diamond anvil cell. The pressure coefficient of $\mathrm{InN}$ is determined to be $3.0 \pm 0.1 \mathrm{meV} / \mathrm{kbar}$. A comparison between our results and previously reported theoretical calculations is presented and discussed. Our data and previous experimental results suggest that the pressure coefficients of group III nitride alloys are only weakly dependent on alloy composition. The pressure coefficients determined from PL are significantly smaller than those of the bandgap due to emission associated with highly localized electron states. The absolute deformation potentials of the CBM and VBM are estimated.

The work at the Lawrence Berkeley National Laboratory is supported by the Director, Office of Science, Office of Basic Energy Sciences, Division of Materials Sciences and Engineering of the U.S. Department of Energy under Contract No. DEAC03-76SF00098. The work at Cornell University is supported by ONR under Contract No. N000149910936. 


\section{References:}

[1] T. Inushima, V. V. Mamutin, V. A. Vekshin, S. V. Ivanov, T. Sakon, M. Motokawa, and S. Ohoya; J. Crystal Growth 227-228, 481 (2001).

[2] V. Yu. Davydov, A. A. Klochikhin, R. P. Seisyan, V. V. Emtsev, S. V. Ivanov, F. Bechstedt, J. Furthmüller, H. Harima, A. V. Murdryi, J. Aderhold, O. Semchinova, and J. Graul; phys. stat. sol. B 229, R1 (2002).

[3] J. Wu, W. Walukiewicz, K. M. Yu, J. W. Ager III, E. E. Haller, Hai Lu, William J. Schaff, Yoshiki Saito, and Yasushi Nanishi; Appl. Phys. Lett. 80, 3967 (2002).

[4] Takashi Matsuoka, Hiroshi Okamoto, Masashi Nakao, Hiroshi Harima, and Eiji Kurimoto; Appl. Phys. Lett. 81, 1246 (2002).

[5] Su-Huai Wei, Xiliang Nie, Iskander G. Batyrev, and S. B. Zhang; Phys. Rev. B 67, 165209 (2003).

[6] J. Wu, W. Walukiewicz, K. M. Yu, J. W. Ager III, E. E. Haller, Hai Lu, William J. Schaff; Appl. Phys. Lett. 80, 4741 (2002)

[7] Sangsig Kim, Irving P. Herman, J. A. Tuchman, K. Doverspike, L. B. Rowland, and D. K. Gaskill; Appl. Phys. Lett. 67, 380 (1995).

[8] W. Shan, W. Walukiewicz, E. E. Haller, B. D. Little, J. J. Song, M. D. McCluskey, N. M. Johnson, Z. C. Feng, M. Schurman, and R. A. Stall; J. Appl. Phys. 84, 4452 (1998).

[9] Piotr Perlin, Laila Mattos, Noad A. Shapiro, Joachim Kruger, William S. Wong, Tim Sands, Nathan W. Cheung, and Eicke R. Weber; J. Appl. Phys. 85, 2385 (1999).

[10] T. Suski, H. Teisseyre, S. P. Łepkowski, P. Perlin, H. Mariette, T. Kitamura, Y. Ishida, H. Okumura, and S. F. Cichibu; phys. stat. sol. (b) 235, 225 (2003).

[11] Hisamitsu Akamaru, Akifumi Onodera, Tadashi Endo, Osamu Mishima; J. Phys. Chem. Solids 63, 887 (2002).

[12] H. Lu, W. J. Schaff, J Hwang, H. Wu, G. Koley, and L. F. Eastman, Appl. Phys. Lett. 79, 1489 (2001).

[13] J. S. Blakemore, J. Appl. Phys. 53, R123 (1982).

[14] Y. F. Ng, Y. G. Cao, M. H. Xie, X. L. Wang, and S. Y. Tong; Appl. Phys. Lett. 81, 3960 (2002). 
[15] V. Cimalla, Ch. Förster, G. Kittler, I. Popa, R. Kosiba, G. Ecke, O. Ambacher, R. Goldhahn, S. Shokhovets, A. Georgakilas, H. Lu, and W. Schaff, phys. stat. sol (a) 195, 3-10 (2003)

[16] Tae-Bong Hur, Ik Jae Lee, Jin Woo Kim, Yoon-Hwae Hwang, and Hyung-Kook Kim, Jpn. J. Appl. Phys. 41, 1932 (2002)

[17] Su-Huai Wei and Alex Zunger, Phys. Rev. B 60, 5404 (1999).

[18] N. E. Crhistensen and I. Gorczyca, Phys. Rev. B 50, 4397 (1994).

[19] G. Martinez, Handbook on Semiconductors Vol. 2, 4c, edited by T. S. Moss, NorthHolland Publishing Co. (1980).

[20] J. C. Phillips, Bonds and Bands in Semiconductors, Academic Press, New York (1973).

[21] Sadao Adachi, J. Appl. Phys. 58, R1 (1985).

[22] D. D. Nolte, W. Walukiewicz, and E.E. Haller; Phys. Rev. Lett. 59, 501 (1987)

[23] J. Wu, W. Walukiewicz, K.M. Yu, J.W. Ager III, E.E. Haller, I. Miotkowski, A. K. Ramdas, and S. Miotkowska; Appl. Phys. Lett. 80, 34 (2002).

[24] W. Walukiewicz, Physica B 302-303, 123 (2001)

[25] The concept has been recently applied to show that the hydrogen levels in semiconductors are constant on an absolute scale, Chris Van de Walle and J. Neugebauer, Nature 423, 626 (2003)

[26] G. Martin, A. Botchkarev, A. Rockett, and H. Morkoç; Appl. Phys. Lett. 68, 2541 (1996)

[27] Numerical Data and Functional Relationships in Science and Technology, edited by O. Madelung and M. Schulz, Landolt-Bornstein, New Series, Group III, Vol. 22 Pt. a, Springer-Verlag, Berlin (1987). 


\section{Figure Captions}

Fig.1 Absorption curves (linear absorption coefficients squared) of $\mathrm{InN}$ taken at room temperature for different hydrostatic pressures.

Fig.2 Bandgap energies as a function of pressure. The straight lines show least square linear fits. The pressure dependence of the PL peak of two samples is also shown. The PL data of InN are from ref [3].

Fig.3 Pressure coefficients of group III nitride alloys. Results of previous experiments $[3,8,10,11]$ and calculations $[17,18]$ are also shown for comparison. (Cal = calculation, Abs $=$ optical absorption, $\mathrm{PL}=$ photoluminescence)

Fig.4 PL energies as a function of Ga composition on the absolute energy scale. The conduction band and valence band offset between $\mathrm{InN}$ and $\mathrm{GaN}$ is taken into account. Shown in the insert are the actual PL spectra of alloys with various Ga compositions. 
Figures

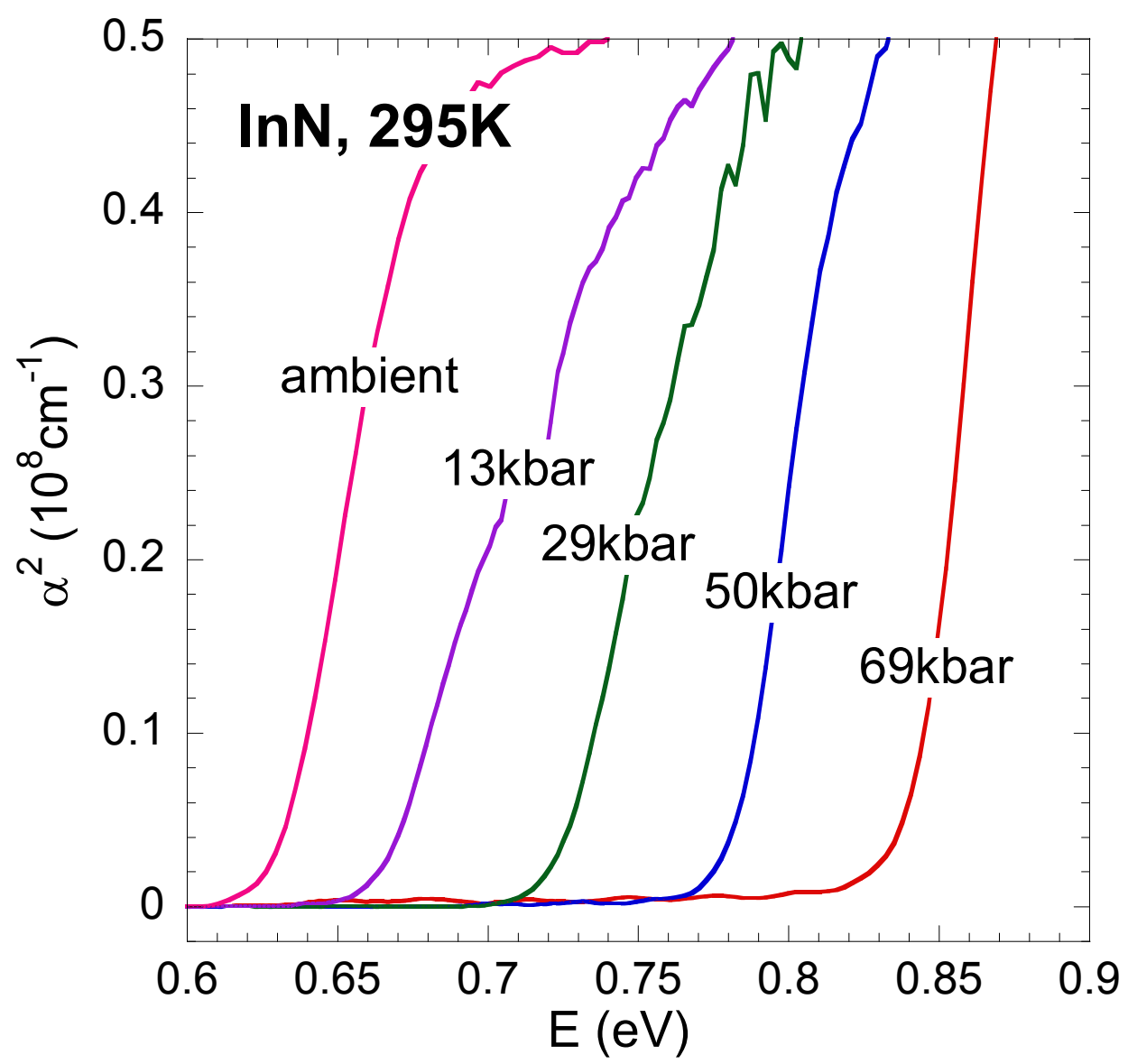

Fig. 1 of 4

Li et. al. 


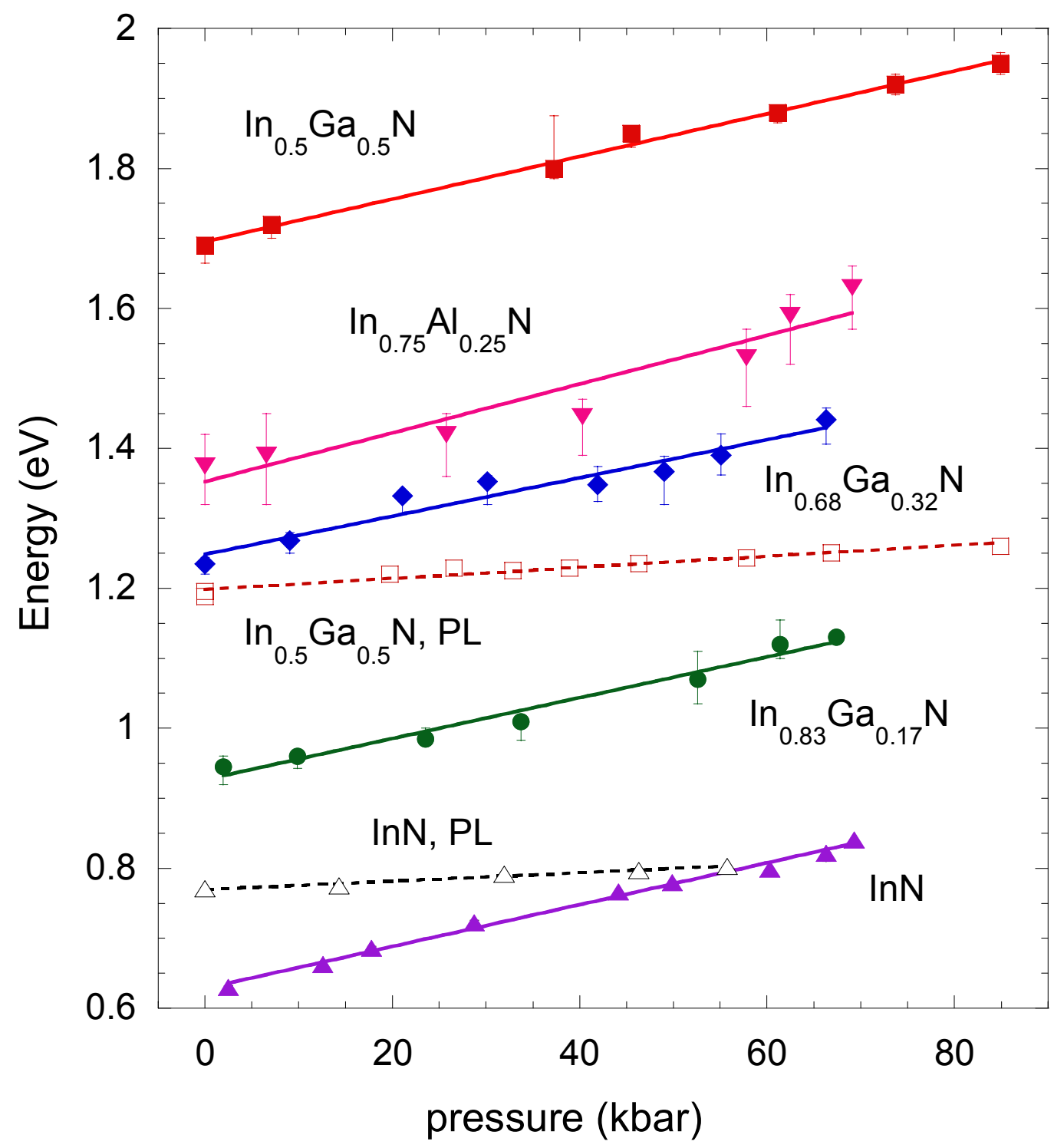

Fig. 2 of 4

Li et. al. 


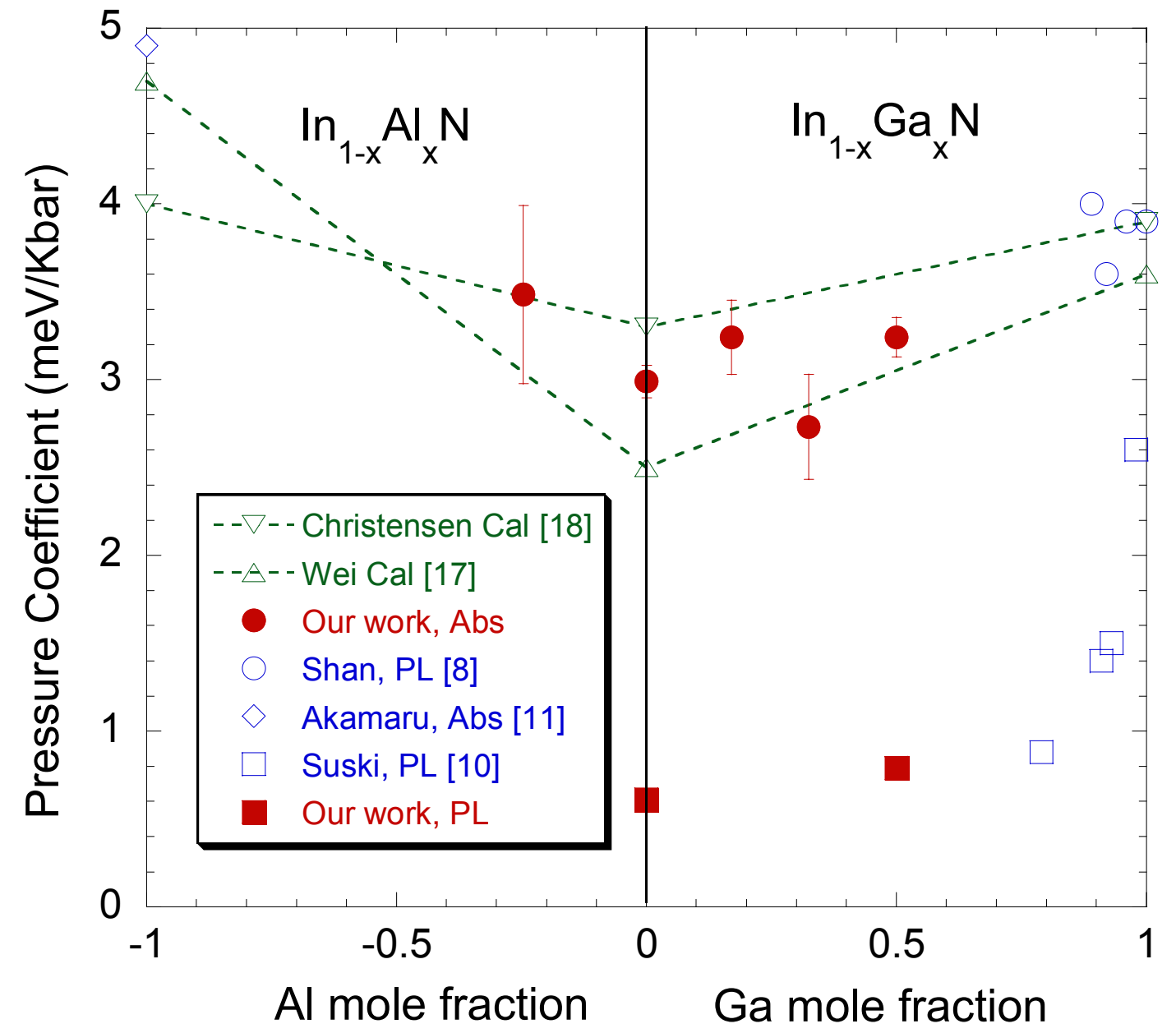

Fig. 3 of 4

Li et. al. 


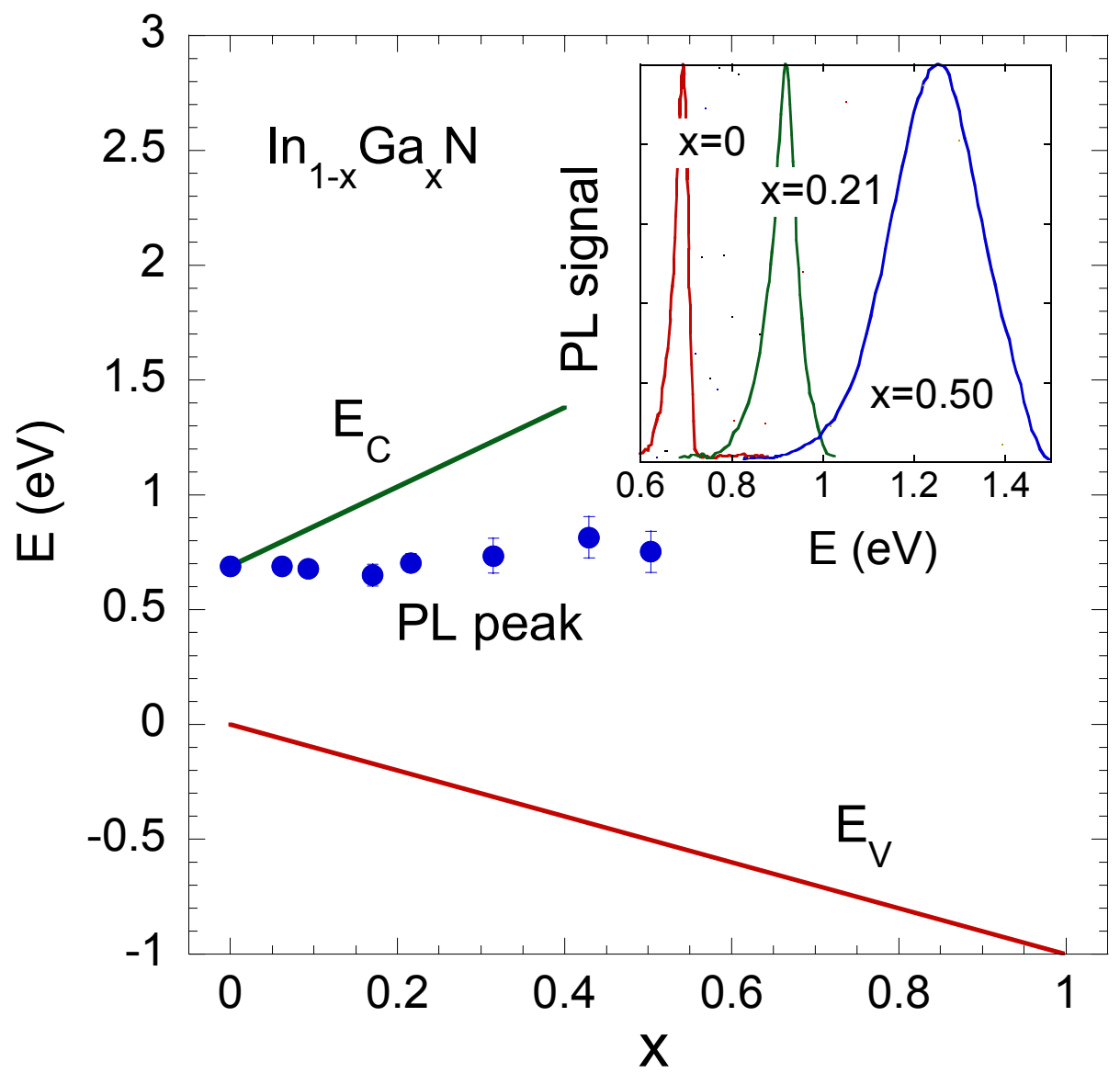

Fig. 4 of 4

Li et. al. 